\title{
Asymmetrical Modulation for Uplink Communication in Cooperative Networks
}

\author{
Zhang, Qi; Fitzek, Frank H.P.; Iversen, Villy Bæk
}

Published in:

Proceeding of ICC workshops

Link to article, DOI:

10.1109/ICCW.2008.21

Publication date:

2008

Document Version

Publisher's PDF, also known as Version of record

Link back to DTU Orbit

Citation (APA):

Zhang, Q., Fitzek, F. H. P., \& Iversen, V. B. (2008). Asymmetrical Modulation for Uplink Communication in Cooperative Networks. In Proceeding of ICC workshops (pp. 85-90). IEEE.

https://doi.org/10.1109/ICCW.2008.21

\section{General rights}

Copyright and moral rights for the publications made accessible in the public portal are retained by the authors and/or other copyright owners and it is a condition of accessing publications that users recognise and abide by the legal requirements associated with these rights.

- Users may download and print one copy of any publication from the public portal for the purpose of private study or research.

- You may not further distribute the material or use it for any profit-making activity or commercial gain

- You may freely distribute the URL identifying the publication in the public portal

If you believe that this document breaches copyright please contact us providing details, and we will remove access to the work immediately and investigate your claim. 


\section{Asymmetrical Modulation for Uplink Communication in Cooperative Networks}

\author{
Qi Zhang \\ Department of Communication, \\ Optics \& Materials \\ Technical University of Denmark \\ Lyngby, Denmark \\ Email: qz@com.dtu.dk
}

\author{
Frank H.P. Fitzek \\ Department of Communications \\ Technology \\ Aalborg University \\ Aalborg, Denmark \\ Email: ff@es.aau.dk
}

\author{
Villy B. Iversen \\ Department of Communication, \\ Optics \& Materials \\ Technical University of Denmark \\ Lyngby, Denmark \\ Email:vbi@com.dtu.dk
}

\begin{abstract}
In this paper a spectrum efficient uplink communication method for cooperative wireless networks is presented. In cellular controlled peer to peer (CCP2P) cooperative wireless networks the mobile device is logically connected over cellular links with the base station and simultaneously over shortrange links with neighboring mobile devices to form cooperative clusters. So far the physical communication over cellular links and over short-range links are separated in time or in frequency. Beyond this state of the art, we exploit a method, referred to as asymmetrical modulation, where a mobile device is generating signals that are conveyed towards the base station and the neighboring devices in the same frequency and even at the same time. The signal is composed in such a way that it has different meanings for the neighboring devices than the base station. While the base station is getting the coarse information, the neighboring devices are getting the fine grained information reading between the lines. Our analytical analysis and simulation results show that asymmetrical modulation can improve the spectrum efficiency and reduce the data queuing delay with neither degrading the symbol error rate performance nor increasing the average energy per bit.
\end{abstract}

\section{INTRODUCTION AND STATE OF THE ART}

Recently cooperative wireless networks have gained more attention. Especially the so called cellular controlled peer to peer (CCP2P) communication architecture [1], [2] has been proven to support higher data rates and at the same time reduces the energy consumption of a mobile device. In CCP2P, mobile devices are not only connected to the base station, but also with the neighboring mobile devices to form cooperative clusters. CCP2P combines the advantage of cellular networks, as the main access to services, and peer to peer networks exploiting wireless grid techniques for efficient usage of the build-in entities such as battery, memory, wireless data rate, etc. So far the cellular and the short-range communication is realized in different frequencies or at different time slots. An example of the separation in frequency is the multi-modal approach, where a mobile device can use 3G, WLAN or DVB$\mathrm{H}$ links for the cellular link and Bluetooth or WLAN for the short-range link as introduced in [4], [3], [5]. When the shortrange link (SRL) and the cellular link (CL) are using the same frequency, they are separated in time [6]. Whatever solution has been found so far is separating the cellular and shortrange communication by orthogonal communication channels realized by separation in frequency, time, or code.

In contrast to that, in this paper an approach is introduced which is clearly beyond this state of the art, transmitting information from a dedicated mobile device to the base station and simultaneously to the neighboring mobile devices at the same time in the same frequency. In a nutshell, one dedicated mobile device is conveying an information signal such that it will be read by the different receiving entities in a different manner.

As given in Fig. 1, the integrated signal conveyed from the originating mobile device consists of two information parts, namely the coarse information part for base station and the fine grained information part for neighboring mobile devices, respectively. As the integrated signal reaching the base station is impacted more by path loss than the short-range connection, the fine grained information part, intended for the neighboring mobile devices is washed out. On the other hand, the neighboring devices can get both information parts in the integrated signal and will subtract the coarse information for the base station to retrieve their relevant information part. In summary, in our approach we exploit the wireless channel with its path loss nature as some kind of native filter to realize the novel communication method.

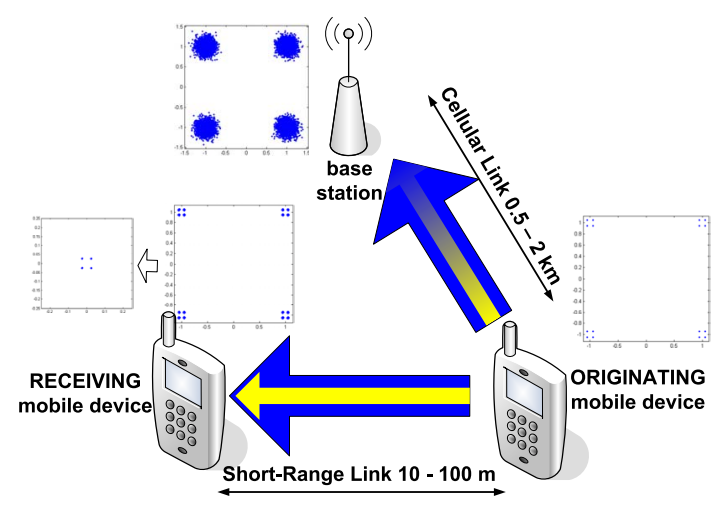

Fig. 1. The applicable network architecture of asymmetrical modulation

The proposed approach is realized by using asymmetrical modulation. Asymmetrical modulation is also called hierar- 
chical modulation which is originally proposed as an unequal error protection technique for extending the service availability and obtaining graceful degradation characteristics [8], [7], [13], [9]. It is mostly used for terrestrial broadcasting of television signals or image transmission, for example HDTV and DVB-H. Asymmetrical modulation is well suited for video transmission over wireless channels, as video encoder outputs are not equally important [9]. It is also employed in multi-class data transmission, when different kinds of data services have different bit error rate requirements [13]. Generally speaking there are two main approaches to implement asymmetrical modulation. The first approach is based on novel signal constellation with non-uniformly spaced signal points [9]. The second approach uses time division multiplexing of different conventional coded modulation schemes [9]. We use the first approach to implement asymmetrical modulation in this paper. All of the proposed asymmetrical modulations [8], [7], [13], [9] are only used for the transmission from BS to mobile devices for unequal error protection purpose. However in our work the asymmetrical modulation is applied for the transmission from a mobile device to the BS and the other mobile devices, i.e., two different types of recipients, with the same error protection.

\section{Asymmetrical Modulation Model \& PERFORMANCE ANALYSIS}

The design idea of asymmetrical modulation (AMOD) for CCP2P is based on the fact that the cellular link (CL) suffers much more path loss than the short-range link (SRL). If we integrate the signals to neighboring mobile devices into the signals to the base station (BS), the path loss on the cellular link can easily filter out all the fine grained information for the neighboring mobile devices. On the other hand, because of the small path loss, the neighboring mobile device can easily extract its own information from the integrated signals. For the illustration purpose, in the following example we assume that the mobile device uses 4QAM to communicate with both, the BS and neighboring mobile devices. It is worth mentioning that for other AMOD constellations the derivation can be done in a similar way as shown later.

Fig. 2 illustrates the basic idea of the AMOD approach. The signal point position of AMOD depends on both the signal to the $\mathrm{BS}$ and the corresponding signal for the neighboring mobile device. The 4QAM signals to the neighboring mobile devices regard the corresponding signal point position for the BS as the origin of its own signal constellation plane (i.e., sub-constellation plane of AMOD). For example, we assume that 4QAM signals for BS $(00,01,10,11)$ are mapped to the four big dashed points $\left(N_{0}, N_{1}, N_{2}, N_{3}\right)$ in the traditional modulation. Therefore, when a signal to the BS is "10" and a signal to the neighboring mobile device is "00" (mapping to the relative Quadrant II in sub-constellation plane around point $N_{2}$ ), the AMOD signal $(1000)$ finally locates its position at the point $M_{0}$.

The MQAM (multiple quadrature amplitude modulation) signal is generically expressed by $S(t)=A \cdot g(t) \cdot e^{j 2 \pi f_{c} t} \cdot g(t)$

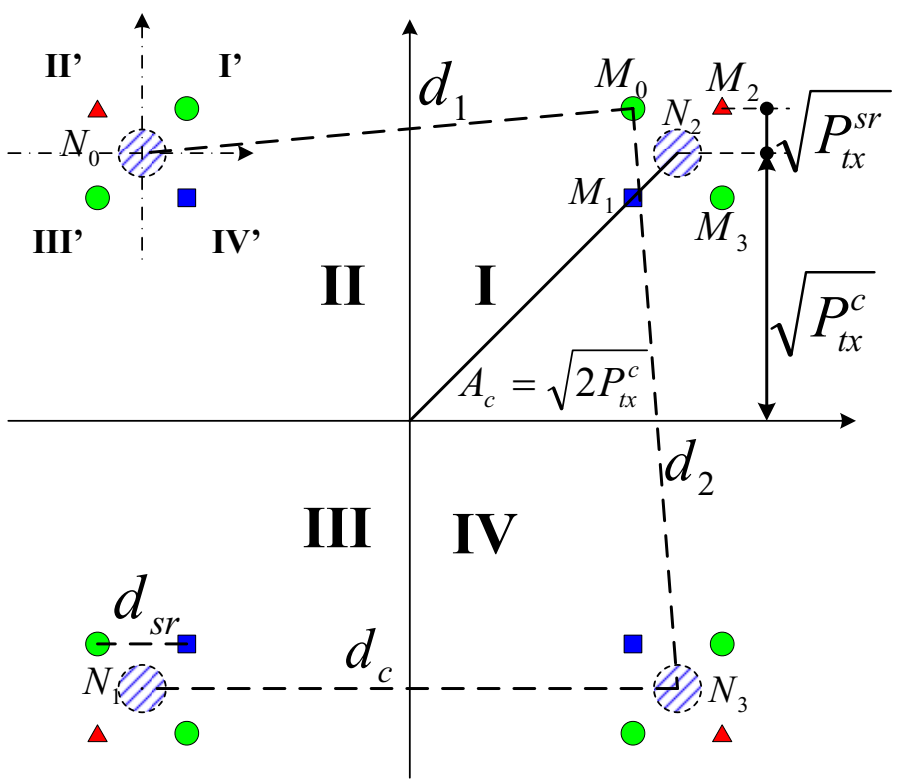

Fig. 2. Asymmetrical modulation constellation at mobile device transmitter

TABLE I

PARAMETER LIST

\begin{tabular}{|c|l|}
\hline Notation & Meaning \\
\hline$d_{i j}$ & $\begin{array}{l}\text { the Euclidean distance between two received signal } \\
\text { points } i \text { and } j \\
\text { the one-sided spectral density of white Gaussian }\end{array}$ \\
$N_{o}$ & $\begin{array}{l}\text { noise } \\
\text { the total number of signal points in a constellation } \\
\text { the average transmission power at mobile device on } \\
\text { the cellular link in traditional modulation }\end{array}$ \\
$P_{t x}^{c}$ & $\begin{array}{l}\text { the average transmission power at mobile device on } \\
\text { the short-range link in traditional modulation } \\
P_{t x}^{s r}\end{array}$ \\
$P_{i}$ & $\begin{array}{l}\text { the mobile device power consumption at idle mode } \\
\text { the fading factor on the CL because of path loss } \\
f_{c} \\
f_{s r}\end{array}$ \\
\hline
\end{tabular}

is the signal pulse with unit amplitude. The average power of the signal is equal to $A^{2} / 2$. Hence, we can assume that the amplitude of the transmission signal on the cellular link is $A_{c}=\sqrt{2 P_{t x}^{c}}$ and on the short-range link $A_{s r}=\sqrt{2 P_{t x}^{s r}}$, respectively. Obviously, using traditional 4QAM the decision regions are bounded by horizontal and vertical lines at zero for both CL and SRL, which does not depends on the amplitude of the signal. But in case of AMOD the decision regions are bounded by horizontal and vertical lines at zero for CL and at $\pm \sqrt{P_{t x}^{c}}$ for SRL.

\section{A. Symbol Error Probability}

In the following we are going to derive the symbol error probability (SER) in the cellular link (CL) and the short-range link (SRL). All of the notations are listed in Table I.

The symbol error probability that a signal which is transmitted as point $i$ is received and demodulated as a point $j$ can be expressed as following:

$$
P_{e, i j}=Q\left(\frac{d_{i j} / 2}{\sqrt{N_{o} / 2}}\right) .
$$


Hence, we may obtain an upper bound for the total symbol error probability as

$$
P_{e} \leq \frac{1}{M} \sum_{i} \sum_{j \neq i} Q\left(\frac{d_{i j} / 2}{\sqrt{N_{o} / 2}}\right) .
$$

Since the $Q$ function is a very fast decreasing function, it is possible to limit the second summation in Eq. 2 to a smaller set of the nearest neighboring points around point $i$. Based on the parameters shown in Fig. 2, we can express the symbol error probability of the received signals at the BS using traditional modulation as following,

$$
P_{e, B S}^{o l d}=2 \cdot Q\left(\frac{d_{c}^{r x} / 2}{\sqrt{N_{o} / 2}}\right)=2 \cdot Q\left(f_{c} \sqrt{\frac{2 P_{t x}^{c}}{N_{o}}}\right),
$$

where, the ideal Euclidean distance of two received signal points at BS, $d_{c}^{r x}$, has such relation that $d_{c}^{r x}=f_{c} d_{c}=$ $2 f_{c} \sqrt{P_{t x}^{c}}$, which is clear from Fig. 2.

Using AMOD in this specific example, there are 16 possible signal points which can be divided into three types (see Fig. 2): (i) $\square$ (ii) $\bigcirc$ and (iii) $\triangle$, according to their positions in the constellation. Signal points at different position have different probabilities to make an error to the nearest neighboring points. We assume that at the BS receiver the probability that point $M_{0}$ is demodulated as $N_{0}$ and $N_{3}$ is $p_{1}$ and $p_{2}$, respectively. Therefore, the error probability of $M_{0}$ is $p_{1}+p_{2}$, which is same for all $\bigcirc$ type signal points. (Note, in Fig. 2 the BS always supposes that the signal points from mobile device are at the big dashed points, although the real signal points are located at solid points.)

$$
p_{1}=Q\left(\frac{d_{1}^{r x} / 2}{\sqrt{N_{o} / 2}}\right), \quad p_{2}=Q\left(\frac{d_{2}^{r x} / 2}{\sqrt{N_{o} / 2}}\right) .
$$

At the BS receiver the symbol error probability of point $\square$, such as $M_{1}$, is $2 p_{1}$ and the error probability of point $\triangle$, such as $M_{2}$, is $2 p_{2}$. So the average symbol error probability of received signals at the BS by asymmetrical modulation can now be calculated by:

$$
\begin{aligned}
P_{e, B S}^{a m o d} & =\frac{1}{16}\left\{4 \cdot 2 p_{1}+8 \cdot\left(p_{1}+p_{2}\right)+4 \cdot 2 p_{2}\right\} \\
& =Q\left(\frac{d_{1}^{r x} / 2}{\sqrt{N_{o} / 2}}\right)+Q\left(\frac{d_{2}^{r x} / 2}{\sqrt{N_{o} / 2}}\right),
\end{aligned}
$$

where, according to Fig. 2 and considering signal attenuation, the Euclidean distance of the received signals at the BS receiver $d_{1}^{r x}$ and $d_{2}^{r x}$ can be derived as

$$
\begin{aligned}
d_{1}^{r x} & =f_{c} d_{1} \\
& =f_{c} \sqrt{\left(\sqrt{2 P_{t x}^{c}}-\sqrt{P_{t x}^{s r}}\right)^{2}+\left(\sqrt{P_{t x}^{s r}}\right)^{2}} \\
& =f_{c} \sqrt{4 P_{t x}^{c}-4 \sqrt{P_{t x}^{c} P_{t x}^{s r}}+2 P_{t x}^{s r}} .
\end{aligned}
$$

Assuming $\sqrt{P_{t x}^{s r}}=\sigma \sqrt{P_{t x}^{c}}(\sigma \ll 1)$, then

$$
d_{1}^{r x}=f_{c} \sqrt{2 P_{t x}^{c}} \sqrt{2-2 \sigma+\sigma^{2}} .
$$

Likewise, we can obtain expression of $d_{2}^{r x}$,

$$
d_{2}^{r x}=f_{c} \sqrt{2 P_{t x}^{c}} \sqrt{2+2 \sigma+\sigma^{2}} .
$$

For $x>3$, the $Q(x)$ can be approximated as

$$
Q(x) \approx \frac{1}{\sqrt{2 \pi} x} e^{-\frac{x^{2}}{2}} \quad x>3 .
$$

Therefore, according to Eq. 5 we can rewrite $P_{e, B S}^{o l d}$ and $P_{e, B S}^{a m o d}$ as following:

$$
\begin{gathered}
P_{e, B S}^{o l d} \approx \frac{1}{f_{c}} \sqrt{\frac{N_{o}}{\pi P_{t x}^{c}}} e^{-\frac{f_{c}^{2} P_{t x}^{c}}{N_{o}}}, \\
P_{e, B S}^{h m o d} \approx \frac{1}{f_{c}} \sqrt{\frac{N_{o}}{2 \pi P_{t x}^{c}}}\left(\frac{1}{\sqrt{\gamma_{1}}} e^{-\frac{f_{c}^{2} P_{t x}^{c} \gamma_{1}}{2 N_{o}}}+\frac{1}{\sqrt{\gamma_{2}}} e^{-\frac{f_{c}^{2} P_{t x}^{c} \gamma_{2}}{2 N_{o}}}\right)
\end{gathered}
$$

with

$$
\gamma_{1}=2-2 \sigma+\sigma^{2}, \quad \gamma_{2}=2+2 \sigma+\sigma^{2} .
$$

When $\sigma \ll 1, \gamma_{1}$ and $\gamma_{2}$ are approximately equal to 2 . By substituting the approximate values of $\gamma_{1}$ and $\gamma_{2}$ into Eq. 6, we obtain Eq. 7. It can be seen from Eq. 7 that asymmetrical modulation can achieve the same symbol error probability as the traditional modulation on the cellular link.

$$
P_{e, B S}^{a m o d} \approx \frac{1}{f_{c}} \sqrt{\frac{N_{o}}{\pi P_{t x}^{c}}} e^{-\frac{f_{c}^{2} P_{t x}^{c}}{N_{o}}} \approx P_{e, B S}^{o l d}
$$

As for the symbol error probability on the short-range link, it is derived as following.

$$
P_{e, s r}^{h m o d}=1-\left(1-P_{e, 1}\right)\left(1-P_{e, 2}\right)
$$

here, $P_{e, 1}$ is the symbol error probability of the signal for base station at the demodulator of the cooperative mobile device, and $P_{e, 2}$ is the symbol error probability of the signal for the cooperative mobile device at the demodulator of the operative mobile device.

It is clear that $P_{e, s r}^{o l d}$ is equal to $P_{e, 2}$

$$
P_{e, s r}^{o l d}=P_{e, 2} \leq 2 Q\left(\frac{d_{s r}^{r x} / 2}{\sqrt{N_{o} / 2}}\right)
$$

where, Euclidean distance of the received signals at the neighboring mobile device receiver, $d_{s r}^{r x}$, is expressed by

$$
d_{s r}^{r x}=f_{s r} d_{s r}=2 f_{s r} \sqrt{P_{t x}^{s r}} .
$$

Because $P_{e, 1}$ in Eq. 8 is close to zero in the application scenario described in this paper, there is:

$$
P_{e, s r}^{h m o d} \approx P_{e, s r}^{o l d} \leq 2 Q\left(f_{s r} \sqrt{\frac{2 P_{t x}^{s r}}{N_{o}}}\right)
$$

\section{B. Average Energy Per Bit}

In this subsection, the average energy per bit, $E_{b}$, and the average energy consumption is derived for both modulation schemes. We assume that the cellular link and the shortrange link use 4QAM and the same amount of data has to be transmitted. The baud rate is $\frac{1}{T}$ symbol/s. Therefore, in traditional modulation the average energy per bit at the transmitting mobile device can be expressed as

$$
E_{b}^{o l d}=\frac{1}{2}\left(\frac{E_{s}^{c}+E_{s}^{s r}}{2}\right),
$$


where $E_{s}^{c}$ and $E_{s}^{s r}$ is the energy per symbol on the cellular link and short-range link. They can be expressed by

$$
\begin{gathered}
E_{s}^{c}=\int_{0}^{T}\left(\sqrt{2 P_{t x}^{c}} \cos \left(2 \pi f_{c} t+\theta\right)\right)^{2} d t=P_{t x}^{c} T \\
E_{s}^{s r}=\int_{0}^{T}\left(\sqrt{2 P_{t x}^{s r}} \cos \left(2 \pi f_{c} t+\theta\right)\right)^{2} d t=P_{t x}^{s r} T,
\end{gathered}
$$

Therefore,

$$
E_{b}^{o l d}=\frac{1}{4}\left(P_{t x}^{c}+P_{t x}^{s r}\right) T .
$$

The asymmetrical modulation in this example becomes an asymmetrical 16QAM. To transmit the same amount of data, the baud rate is the same with $\frac{1}{T}$ symbol/s. But each symbol has 4 bits. So the average energy per bit, $E_{b}$, can be expressed as following, considering the probability of different signals' position.

$$
E_{b}^{\text {amod }}=\frac{1}{4} E_{s}^{\text {amod }}
$$

where, $E_{s}^{\text {amod }}$ is the energy per symbol of the asymmetrical modulation signal. Due to the instant energy per symbol depending on signal point position, the signal's amplitude can be categorized as $A_{0}, A_{1}$ and $A_{2}$ for signal point $\square, \bigcirc$ and $\triangle$, respectively. So the average energy per symbol $E_{s}^{\text {amod }}$ can be simply given by

$$
\begin{aligned}
E_{s}^{a \text { amod }}= & \frac{1}{16}\left(4 \int_{0}^{T}\left(A_{0} g(t) \cos \left(2 \pi f_{c} t+\theta_{0}\right)\right)^{2} d t\right)+ \\
& \frac{1}{16}\left(8 \int_{0}^{T}\left(A_{1} g(t) \cos \left(2 \pi f_{c} t+\theta_{1}\right)\right)^{2} d t\right)+ \\
& \frac{1}{16}\left(4 \int_{0}^{T}\left(A_{2} g(t) \cos \left(2 \pi f_{c} t+\theta_{2}\right)\right)^{2} d t\right)
\end{aligned}
$$

where, letting $g(t)=1$, furthermore, $\theta_{0}=\theta_{2}=\frac{\pi}{4}, \theta_{1}=$ $\tan ^{-1} \frac{\sqrt{P_{t x}^{c}}-\sqrt{P_{t x}^{s r}}}{\sqrt{P_{t x}^{c}}+\sqrt{P_{t x}^{s r}}}$ and $f_{c} \gg \frac{1}{T}$

where,

$$
\begin{aligned}
& A_{0}=\sqrt{2}\left(\sqrt{P_{t x}^{c}}-\sqrt{P_{t x}^{s r}}\right) \\
& A_{1}=\sqrt{\left(\sqrt{P_{t x}^{c}}+\sqrt{P_{t x}^{s r}}\right)^{2}+\left(\sqrt{P_{t x}^{c}}-\sqrt{P_{t x}^{s r}}\right)^{2}} \\
& A_{2}=\sqrt{2}\left(\sqrt{P_{t x}^{c}}+\sqrt{P_{t x}^{s r}}\right) .
\end{aligned}
$$

Substituting $A_{0}, A_{1}$ and $A_{2}$ into Eq. 16, we obtain $E_{s}^{a m o d}=$ $\left(P_{t x}^{c}+P_{t x}^{s r}\right) T$. Therefore, there is

$$
E_{b}^{a m o d}=\frac{1}{4}\left(P_{t x}^{c}+P_{t x}^{s r}\right) T .
$$

From the results of Eq. 14 and Eq. 17, it is clear that the average energy per bit is the same for both modulation schemes.

\section{Spectrum Efficiency and Transmission Delay}

From symbol error probability and power consumption performance analysis, we have proven that asymmetrical modulation can achieve the same performance as traditional modulation. The advantage of AMOD is that it increases spectrum efficiency and at the same time it reduces the delay.
In the given example if the spectrum efficiency is $2 \zeta$ bits $/ \mathrm{s} / \mathrm{Hz}$ by traditional modulation then with AMOD the spectrum efficiency is doubled i.e., $4 \zeta$ bits $/ \mathrm{s} / \mathrm{Hz}$. The asymmetrical modulation method that we present here is very flexible. It can have many variations not only 4QAM on both cellular link (CL) and short-range link (SRL). It can be 4QAM on CL and 16QAM on SRL; or it can be 16QAM on CL and BPSK on SRL, 16QAM on CL and 4QAM on SRL, and so on. Which AMOD constellation to use depends on the amount of data flows towards base station and the neighboring mobile device. It also depends on the wireless link quality. So the achievable spectrum efficiency gain depends on the AMOD constellation.

As for the data queuing delay due to waiting for time slots, it is not difficult to understand that asymmetrical modulation can reduce such delay nearly by half.

In summary, AMOD improves the spectrum efficiency and delay performance, with neither any system performance degradation nor additional costs.

\section{Simulation \& Results}

To validate our analytical findings, simulative measurements were conducted. This section describes the simulation procedure and shows the simulation results. The basic simulation procedure is illustrated in Fig. 3. In the simulation, we are interested in the different effects of different path losses on the received symbols. Therefore, we only consider the signal amplitude attenuation and AWGN (Additive White Gaussian Noise) channel is used in the simulation. Here we can assume that attenuation is a function of the distance between antennas but is constant in the available frequency bandwidth.

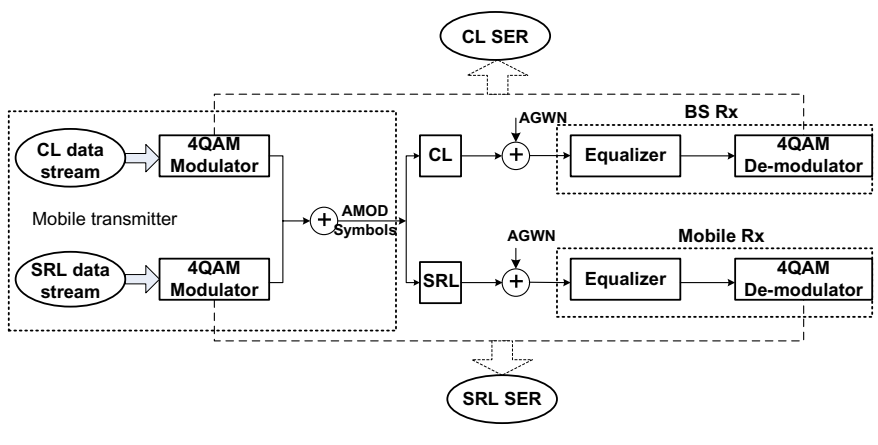

Fig. 3. Simulation chart

The power of the received signal at the BS receiver and at the neighboring mobile device can be expressed as following:

$$
\begin{aligned}
& P_{r x}^{c}=P_{t x}^{c}+G_{m}-L_{b}-P L_{c}+G_{b s}-L_{c b} \\
& P_{r x}^{s r}=P_{t x}^{s r}+G_{m}-L_{b}-P L_{s r}+G_{m}
\end{aligned}
$$

The path loss is calculated based on proper propagation models. Propagation models of the CL and the SRL used in the simulation are given as following. The values of the parameters for the assumption in the simulation are listed in Table II [10], [12], [14]. 
TABLE II

SiMULATION PARAMETER VALUES

\begin{tabular}{|c|l|c|}
\hline Notation & Meaning & Value \\
\hline$f$ & Frequency & $1950 \mathrm{MHz}$ \\
$B$ & Bandwidth & $200 \mathrm{kHz}$ \\
$P_{t x}^{c}$ & Mobile transmission power on CL & $30 \mathrm{dBm}$ \\
$P_{t x}^{s r}$ & Mobile transmission power on SRL & $6.25 \mathrm{e}-4 \mathrm{dBm}$ \\
$G_{m}$ & Mobile antenna gain & $0 \mathrm{dBi}$ \\
$L_{b}$ & Body loss & $0 \mathrm{~dB}$ \\
$E I R P$ & Equivalent Isotropic Radiated Power & $30 \mathrm{dBm}$ \\
$n_{d}$ & Thermal noise density & $-174.0 \mathrm{dBm} / \mathrm{Hz}$ \\
$N_{f}$ & Receiver noise Figure & $7.0 \mathrm{~dB}$ \\
$n_{r x}$ & Receiver noise density & $-167.0 \mathrm{dBm} / \mathrm{Hz}$ \\
$N_{r x}$ & Receiver noise power & $-114.5 \mathrm{dBm}$ \\
$I_{m}$ & Interference margin & $3 \mathrm{dBm}$ \\
$N I$ & Total effective noise and interference & $-111.5 \mathrm{dBm}$ \\
$G_{b s}$ & Base station antenna gain & $18 \mathrm{dBi}$ \\
$L_{c b}$ & Cable loss in the base station & $2 \mathrm{~dB}$ \\
$h_{b s}$ & Base station height & $30 \mathrm{~m}$ \\
$h_{m}$ & Mobile device height & $1.5 \mathrm{~m}$ \\
$\mathrm{PL}_{0}$ & Intercept in SR path loss formula & $38.2 \mathrm{~dB} / 42 \mathrm{~dB}$ \\
$\gamma$ & Path loss exponent & $2 / 2.8$ \\
$S$ & Random scatter about the regression line & $2.8 \mathrm{~dB} / 4.4 \mathrm{~dB}$ \\
$R_{c}$ & Antenna distance in CL & {$[0.5-2] \mathrm{km}$} \\
$R_{s r}$ & Antenna distance in SR & {$[0.01-1] \mathrm{km}$} \\
\hline
\end{tabular}

\section{A. Path Loss of Cellular Link}

To calculate the path loss of cellular link, $P L_{c}$, we use the famous Okumura-Hata propagation model for an urban macro cell with the BS antenna height of $30 \mathrm{~m}$ and mobile antenna height $1.5 \mathrm{~m}$ with carrier frequency of $1950 \mathrm{MHz}$ [15]:

$$
P L_{c}=137.4+35.2 \log _{10}\left(R_{c}\right) \quad(d B),
$$

where, $R_{c}$ is the range in $\mathrm{km}$. For suburban areas it is usually assumed an additional area correction factor of $8 \mathrm{~dB}$ [10].

\section{B. Path Loss of Short-Range Link}

It is quite challenging to calculate the path loss of the short-range link. The typical BS to mobile propagation model cannot be applied to mobile to mobile (M2M) model as: 1.) one antenna is not in the far field of the other antenna [11]. 2.) for low antenna height the effects of the close proximity between the Earth and the antenna produce a strong interaction between the antenna and the ground, and the antenna pattern performance is vastly different than if the antenna is in free space [11].

A comprehensive survey is performed in [11], which studied the available propagation models and measured data in the current 125 literature references to determine their applicability to the short-range M2M propagation model. But unfortunately none of the models fits exactly our scenario. Considering each model's applicability and limitation, we combine the results from two models [12], [14] and apply them to our simulation.

The short-range propagation model given in [12] is used for UWB (Ultra Wide Band) network at frequency $5 \mathrm{GHz}$. And a similar model is given in [14] which is focus on $1.8 \mathrm{GHz}$. The path loss in short-range link, $P L_{s r}$, is given as

$$
P L_{s r}=\left[P L_{0}+10 \gamma \log _{10}\left(R_{s r}\right)\right]+S ; \quad R_{s r}>R_{0},
$$

where, $R_{0}=1 \mathrm{~m}$. The distance between antennas, $R_{s r}$, is in meter; bracketed term is a least-squares fit to path loss. $P L_{0}$ is

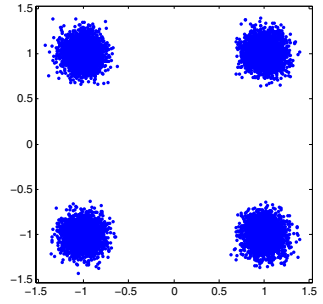

(a) 4QAM/CL, 4QAM/SRL

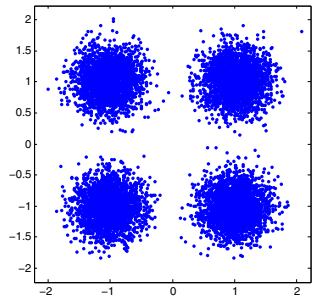

(b) 4QAM/CL, 16QAM/SRL
Fig. 4. Asymmetrical modulated signal constellation at BS receiver, 4QAM on cellular link, 4QAM or 16QAM on short-range link (SRL)

intercept and $\gamma$ is path loss exponent. $S$ is the random scatter about the regression line, assumed to be a zero-mean Gaussian variate with standard deviation $\sigma \mathrm{dB}$. In [12] it gives the mean value of $P L_{0}$. Considering our target frequency, we recalculate mean value of $P L_{0}$ for LOS/NLOS scenarios, (see Table II). The mean value and standard deviation of normal random variants $\gamma$ and $S$ are taken from [12], [14] (see Table II).

\section{Simulation Results Details}

Two scenarios of the asymmetrical modulation are simulated here. One is 4QAM for the cellular link (CL) and the shortrange link (SRL), the other scenario is $4 \mathrm{QAM}$ on CL and 16QAM on SRL.

The AMOD transmission signal gets attenuation and distortion when it passes the CL. At the receiver side, it includes AGWN (Additive Gaussian White Noise) in the signal. The received signals at the BS after equalizer are shown in Fig. 4. It shows that the BS is only able to distinguish the 4QAM signals for itself. Furthermore, the 4QAM modulated signal has good resolution for the BS to demodulate, which is proven by Fig. 5. It shows that the SER of CL in two scenarios is always less than $10^{-6}$ when the antenna distance is less than $1.2 \mathrm{~km}$. Up to antenna distance $1.55 \mathrm{~km}$, the SER is always below $10^{-3}$. It also shows that AMOD has almost the same SER performance as traditional modulation on the cellular link, although when using 16QAM on the SRL, the SER of AMOD is a little bit higher than that of the traditional one. Comparing the AMOD SERs of the two scenarios, it does have slight higher SER when using 16QAM on the SRL. In order to achieve at least $10^{-3}$ SER, the performance difference of the two scenarios is about $50 \mathrm{~m}$ coverage radius. In practice, considering the application needs and wireless channel quality, both constellations are applicable.

Fig. 6 shows the constellation of the received signal at the neighboring mobile side. Due to the lower path loss, it is clear that the received signal is good enough for the mobile to extract the fine grained information. Fig. 7 compares the SERs on the short-range link for both LOS and NLOS cases of the two scenarios. The SER is below $10^{-6}$ when the antenna distance is less than $50 \mathrm{~m}$ for NLOS environment. To have the same SER performance in LOS environment, the antenna distance can be up to $400 \mathrm{~m}$. Furthermore, like theoretical analysis, the SER performance of AMOD is also same as the 


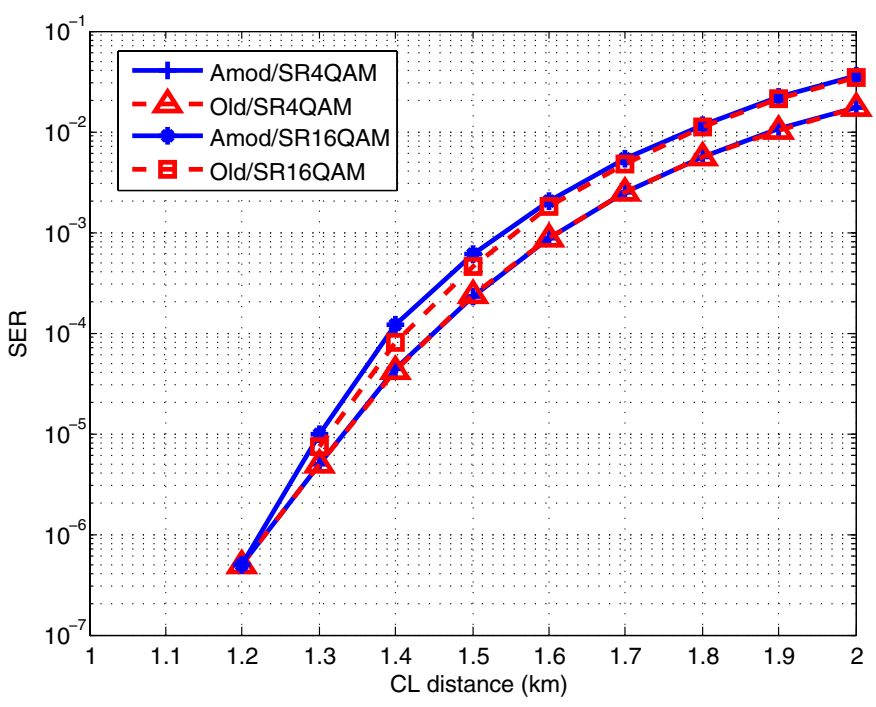

Fig. 5. Symbol Error Rate comparison at the receiver of base station
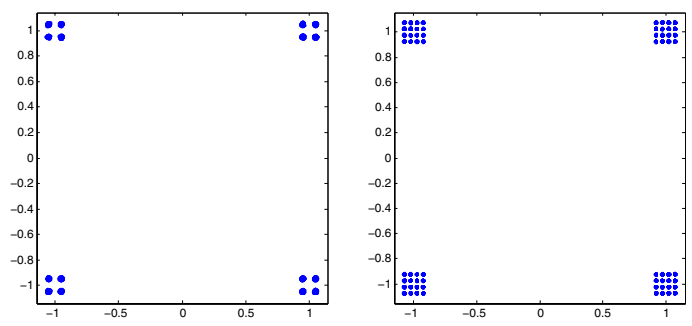

(a) $4 \mathrm{QAM} / \mathrm{CL}, 4 \mathrm{QAM} / \mathrm{SRL} \quad$ (b) $4 \mathrm{QAM} / \mathrm{CL}, 16 \mathrm{QAM} / \mathrm{SRL}$

Fig. 6. Received asymmetrical modulation signal constellation at the neighboring mobile device, 4QAM on cellular link, 4QAM or 16QAM on short-range link (SRL)

traditional modulation on the short-range link. For the scenario using 16QAM on SRL, it has slightly higher SER than the other.

\section{CONCLUSION}

This paper presents a novel method for a mobile device transmitting different signals towards the BS and the neighboring mobile devices in the same frequency and at the same time using asymmetrical modulation. By analytical and simulation results it is proven that the proposed method significantly improves the uplink spectrum efficiency and reduces the data queuing delay with neither degrading the symbol error rate performance nor consuming extra energy. The proposed communication method is targeted to facilitate the signalling and data transmission in cellular controlled peer to peer (CCP2P) communication network architecture.

\section{REFERENCES}

[1] Frank H.P. Fitzek and Marcos D.Katz, editors. Cooperation in Wireless Networks: Principle and Applications. ISBN-10 1-4020-4710-X. 2006.

[2] Frank H.P. Fitzek and Marcos Katz, editors. Cognitive Wireless Networks: Concepts, Methodologies and Visions. ISBN: 978-1-4020-59780. Springer, 1st edition, 2007.

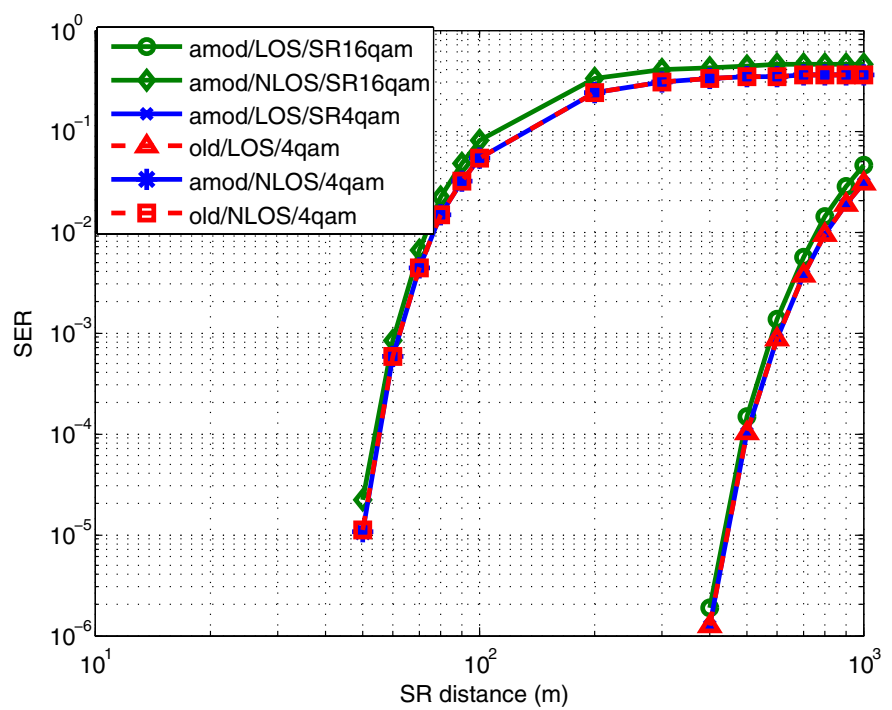

Fig. 7. Symbol Error Rate comparison at the receiver of the neighboring mobile device

[3] Hung-Yun Hsieh and R. Sivakumar. On using peer-to-peer communication in cellular wireless data networks. IEEE Transactions on Mobile Computing, 3(1):57-72, 2004.

[4] Masato YAMADA, Ryoichi SHINKUMA, and Tatsuro TAKAHASHI. Cooperative networking based on infrastructure multihop architecture in heterogeneous mobile networks. IEICE - Transactions on Communications, E89-B(10):2763-2773, 2006.

[5] Qi Zhang, Frank H.P. Fitzek, and Marcos Katz. Cooperative power saving strategies for ip-services supported over dvb-h networks. In Wireless communication\&network conference, March 2007.

[6] Qi Zhang, Frank H.P. Fitzek, and Villy B. Iversen. Design and performance evaluation of cooperative retransmission scheme for reliable multicast services in cellular controlled p2p networks. In The 18th Annual IEEE International Symposium on Personal, Indoor and Mobile Radio Communications (PIMRC07), September 2007.

[7] Mohamed M. Khairy and Evaggelos Geraniotis. Asymmetric modulation and multistage coding for multicasting with multi-level reception over fading channels. Proceedings - IEEE Military Communications Conference MILCOM, 1:92-96, 1999.

[8] J.-H. Park and C.W. Lee. Asymmetric 8-psk modulation with embedded bpsk. Electronics Letters, 30(17):1376-1378, 1994.

[9] L.-F. Wei. Coded modulation with unequal error protection. IEEE Transactions on Communications, 41(10):1439-1449, 1993.

[10] Harri Holma and Antti Toskala, editors. WCDMA for UMTS, Radio Access for Third Generation Mobile Communications. John Wiley \& Sons, 2nd edition, 2002.

[11] Nicholas DeMinco. Propagation loss prediction considerations for closein distances and low-antenna height applications. Technical Report NTIA Report TR-07-449, Institute for Telecommunication Science (ITS) of National Telecommunications and Information Administration (NTIA), http://www.its.bldrdoc.gov/pub/ntia-rpt/07-449/07-449.pdf, July 2007.

[12] S. Ghassemzadeh and V. Tarokh. The ultra-wideband indoor path loss model. In IEEE P802.15-02/277-SG3a and IEEE P802.15-02/278-SG3a. July 2002.

[13] Md. Jahangir Hossain, P.K. Vitthaladevuni, M.-S. Alouini, V.K. Bhargava, and A.J. Goldsmith. Adaptive hierarchical modulation for simultaneous voice and multiclass data transmission over fading channels. IEEE Transactions on Vehicular Technology, 55(4):1181-1194, 2006.

[14] N. Patwari, G.D. Durgin, T.S. Rappaport, and R.J. Boyle. Peer-to-peer low antenna outdoor radio wave propagation at $1.8 \mathrm{ghz}$. Vehicular Technology Conference, 1999 IEEE 49th, 1:371-375, 1999.

[15] S. Saunders. Antennas and Propagation for Wireless Communication System. John Wiley \& Sons, 1999. 